\title{
Neuropeptide $Y$ induces ischemic angiogenesis and restores function of ischemic skeletal muscles
}

\author{
Edward W. Lee, ${ }^{1}$ Mieczyslaw Michalkiewicz, ${ }^{2}$ Joanna Kitlinska, ${ }^{1}$ Ivana Kalezic, ${ }^{3}$ \\ Hanna Switalska, ${ }^{4}$ Peter Yoo, ${ }^{1}$ Amarin Sangkharat,${ }^{1}$ Hong Ji, ${ }^{1}$ Lijun Li, ${ }^{1}$ \\ Teresa Michalkiewicz, ${ }^{2}$ Milos Ljubisavljevic, ${ }^{3}$ Hakan Johansson, ${ }^{3}$ Derrick S. Grant, ${ }^{5}$ \\ and Zofia Zukowska ${ }^{1}$
}

\begin{abstract}
${ }^{1}$ Department of Physiology and Biophysics, Georgetown University Medical Center, Washington, DC, USA
${ }^{2}$ Department of Physiology, Medical College of Wisconsin, Milwaukee, Wisconsin, USA

${ }^{3}$ Center for Musculoskeletal Research, Umea, Sweden

${ }^{4}$ Department of Hypertension and Angiology, Warsaw Medical Academy, Warsaw, Poland

${ }^{5}$ Cardeza Foundation for Hematological Research, Jefferson University, Philadelphia, Pennsylvania, USA
\end{abstract}

Previously we showed that neuropeptide Y (NPY), a sympathetic vasoconstrictor neurotransmitter, stimulates endothelial cell migration, proliferation, and differentiation in vitro. Here, we report on NPY's actions, receptors, and mediators in ischemic angiogenesis. In rats, hindlimb ischemia stimulates sympathetic NPY release (attenuated by lumbar sympathectomy) and upregulates NPY-Y2 (Y2) receptor and a peptidase forming Y2/Y5-selective agonist. Exogenous NPY at physiological concentrations also induces $\mathrm{Y} 5$ receptor, stimulates neovascularization, and restores ischemic muscle blood flow and performance. NPY-mediated ischemic angiogenesis is not prevented by a selective Y1 receptor antagonist but is reduced in $\mathrm{Y2}^{-/-}$mice. Nonischemic muscle vascularity is also lower in $\mathrm{Y2}^{-/-}$mice, whereas it is increased in NPY-overexpressing rats compared with their WT controls. Ex vivo, NPYinduced aortic sprouting is markedly reduced in $\mathrm{Y2}^{-/-}$aortas and spontaneous sprouting is severely impaired in $\mathrm{NPY}^{-/-}$mice. NPY-mediated aortic sprouting, but not cell migration/proliferation, is blocked by an antifetal liver kinase 1 antibody and abolished in mice null for eNOS. Thus, NPY mediates neurogenic ischemic angiogenesis at physiological concentrations by activating $\mathrm{Y} 2 / \mathrm{Y} 5$ receptors and eNOS, in part due to release of VEGF. NPY's effectiveness in revascularization and restoring function of ischemic tissue suggests its therapeutic potential in ischemic conditions.

J. Clin. Invest. 111:1853-1862 (2003). doi:10.1172/JCI200316929.

\section{Introduction}

Angiogenesis is a complex multistep process of formation of new vessels that is regulated by numerous growth factors (1). Most known angiogenic factors, such as bFGF (2) and VEGF (3), are derived from mesenchymal cells that are of either vascular or blood-borne origin. Angiogenesis begins with the activation of endothelial cells and proteases, which leads to the degradation of matrix and subsequent endothelial cell migration, proliferation, and differentiation into capillaries (4). Formation of arteries, or arteriogenesis, also requires migration and proliferation of VSMCs (4). While instrumental in tissue growth and development in the prenatal period, in the adult organism physiological angiogenesis is less

Received for publication October 22, 2002, and accepted in revised form April 15, 2003.

Address correspondence to: Edward W. Lee, Department of Physiology and Biophysics, Georgetown University Medical Center, Box 571460, 3900 Reservoir Road, N.W., Washington, DC 20007, USA. Phone: (202) 687-1241; Fax: (202) 687-7407; E-mail: leeew@georgetown.edu.

Conflict of interest: The authors have declared that no conflict of interest exists.

Nonstandard abbreviations used: neuropeptide Y (NPY); NPY-Y1 (Y1); NPY transgenic (NPY-Tg); anti-fetal liver kinase 1 antibody (anti-flk1). active and is limited to certain organs such as the uterus during the menstrual cycle, pregnancy (5), or intensely exercising skeletal muscle (6).

In pathophysiological situations, however, angiogenesis may become reactivated, for example in tumors (7) and retinopathy (8) and in rheumatoid (9), ischemic heart, and peripheral vascular diseases $(10,11)$. Stimulating endogenous ischemia-driven angiogenesis recently became a promising treatment for salvaging ischemic cardiac and skeletal muscles $(12,13)$. Tissue hypoxia stimulates the release of bFGF (2) and VEGF (3) and upregulates the expression of their receptors (14). Consequently, many investigators have explored the therapeutic potential of revascularizing ischemic tissues through local administration of these and other exogenous angiogenic growth factors $(11-13,15-17)$. While often successful in partial therapeutic revascularization in animal models $(2,15-17)$, other factors may be active endogenously as permissive or driving mediators of ischemic angiogenesis. Sympathetic nerves are the major vascular regulatory system and have long been suspected to provide trophic influences on the innervated tissues (18), but their angiogenic potential has not been considered.

Recently, we found that neuropeptide Y (NPY1-36), also referred to as NPY, a sympathetic cotransmitter and vasoconstrictor (19), is a potent growth factor for 
VSMCs (20) and endothelial cells (21) at nonvasoconstrictive concentrations. In vitro, NPY stimulates human endothelial cell adhesion, migration, proliferation, and differentiation into capillaries (21). Human endothelium expresses receptors for NPY-Y1 (Y1) and Y2 as well as the NPY-converting enzyme DPPIV (21). The potency and efficacy of NPY in stimulating angiogenesis in vitro was found to be similar to those of bFGF and VEGF (21).

NPY exerts pleiotropic actions by activating multiple $\mathrm{G}_{\mathrm{i}} / \mathrm{G}_{\mathrm{o}}$-coupled receptors designated Y1-Y5 (22). Y1 is the predominant vascular receptor and the primary one mediating vasoconstriction (19) and VSMC proliferation (20). Conversely, NPY angiogenic activity in vitro is mediated not by $\mathrm{Y} 1$, but by either $\mathrm{Y} 2$ or $\mathrm{Y} 5$ or both receptors (20), and involves NPY's processing to a Y2/Y5-selective agonist, NPY3-36 (23). This conversion of NPY1-36 from a vasoconstrictive Y1 agonist to a nonvasoconstrictive Y2/Y5 agonist occurs endogenously upon cleavage by endothelial DPPIV, which forms NPY3-36 (24).

Having determined in vitro the potent mitogenic and angiogenic activities of NPY on VSMCs and endothelial cells, we then sought to determine its physiological role and mechanisms in angiogenesis in vivo, using a variety of experimental models. Here, we demonstrate for the first time to our knowledge that neuronally released NPY is an endogenous mediator of ischemic angiogenesis via its $\mathrm{Y} 2 / \mathrm{Y} 5$ receptors and NO- and VEGF-dependent pathways.

\section{Methods}

Animals. The following animals were used: Wistar and Sprague-Dawley rats ( $n=8-10$ each, $250-320$ g; Harlan U.S., Indianapolis, Indiana, USA), $\mathrm{Y}^{-/-}$mice $(n=6-8$, 129SV/Y2-/-; AstraZeneca, Molndal, Sweden), $\mathrm{NPY}^{-/-}$ mice (a gift of D. Palmiter, University of Washington, Seattle, Washington, USA), WT 129SV, eNOS ${ }^{-1-}$ and their WT C57/BL mice $(n=6-8$ each; The Jackson Laboratory, Bar Harbor, Maine, USA), and NPY-overexpressing rats $(n=6-8$ Sprague-Dawley rats with the NPY transgene; Medical College of Wisconsin). All protocols were approved by the Animal Care and Use Committees of Georgetown University and the Medical College of Wisconsin, and the Northern Swedish Regional Committee for Ethics in Animal Experiments in accordance with NIH guidelines.

Hindlimb ischemia model. Animals were anesthetized with intraperitoneal pentobarbital (rats, $50 \mathrm{mg} / \mathrm{kg}$; mice, $160 \mathrm{mg} / \mathrm{kg}$ ). In the mouse and rat, the distal femoral artery was ligated and excised. In some rats, the artery was occluded by insertion of an osmotic minipump catheter to intra-arterially infuse $\mathrm{Y} 1$ antagonist (H409/ $22,0.02 \mu \mathrm{mol} / \mathrm{kg} / \mathrm{min}$ for 14 days) (AstraZeneca). This generated arterial plasma concentrations of approximately $0.5 \mu \mathrm{M}$. Previously, the structurally similar Y1 receptor antagonist (BIBP3226; Thomae GmbH, Biberach, Germany) at a similar concentration $(0.5 \mu \mathrm{M})$ blocked $80-90 \%$ of vasoconstriction caused by exoge- nous and endogenous (stress-elicited) NPY at plasma concentrations higher $(10-100 \mathrm{nM})(25,26)$ than those generated by our NPY pellet ( $<10 \mathrm{nM})$. Placebo or NPY (a total of $1 \mu \mathrm{g}$ per pellet delivered over 14 days) was placed in the form of slow-release pellets in the popliteal fossa. Fourteen days later, rats were reanesthetized and aortic and femoral venous blood was collected distally for measurement of NPY immunoreactivity by ELISA (Bechem, Belmont, California, USA). The aorta was exposed and cannulated above the renal arteries, and the vena cava was cut to drain blood from circulation. Sodium nitroprusside $(50 \mu \mathrm{g} / \mathrm{kg}$ intra-arterially) was injected to maximally vasodilate hindlimb vasculature. In some rats, 100,000 Ultraspheres (Interactive Medical Technologies Inc., North Hollywood, California, USA) were injected intra-arterially, and in another group of rats, radiopaque latex (Flow Tech Inc., Boston, Massachusetts, USA) was injected in the same manner. After 15-50 minutes for equilibration, both kidneys and gastrocnemius muscles from both hindlimbs of each rat were harvested. Lumbar (L1-L5) postganglionic sympathectomy was performed surgically on the ischemic side, as described (27).

Electric stimulation of gastrocnemius muscles. Fourteen days after femoral occlusion, rats were reanesthetized and the gastrocnemius muscle and the primary branches of the medial and lateral gastrocnemius nerves were exposed. Submaximal isometric contractions were elicited by electrical stimulation. This was delivered by bipolar platinum electrodes placed on the isolated, distal part of the medial and lateral gastrocnemius nerves. The strength of the stimulus was adjusted to three to five times the motor threshold (0.1-millisecond pulse duration). The gastrocnemius muscle was subjected to repetitive, fatiguing, tetanic stimulations with an increasing number of trains $(1,3,6,12,30$, and 60 , for a total of 112 stimulations at 60 -second intervals) at an $85-\mathrm{Hz}$ fusion frequency (600-millisecond duration, 1-second intervals) in isometric conditions (28). The following measurements were made and compared between groups: peak tetanic force, isometric speed, and fatigability of the muscle (fatigue index). Peak force was defined as the maximum value of tetanic contraction achieved during the first train of $85-\mathrm{Hz}$ stimulation (minus the passive force developed immediately before the beginning of contraction). Isometric speed was defined as the time from the onset of the mechanical response until the attainment of the maximal slope of tetanic contraction. The fatigue index was defined as the ratio of the peak force of the last tetanic contraction at the end of the fifth series (the $52 \mathrm{nd}$ contraction) to that of the first tetanic contraction in the first series (with a greater percent ratio indicating less fatigability).

Immunostaining and measurement of vascularization. Immunostaining with $\mathrm{H} \& \mathrm{E}$, vWF, and $\mathrm{cd} 31$ was performed on paraffin-embedded slides. For vWF staining, rabbit anti-vWF antibody was used as the primary antibody and biotinylated goat anti-rabbit IgG (SigmaAldrich, St. Louis, Missouri, USA) as the secondary 
antibody. Biotin-avidin peroxidase (ABC Kit; Vector Laboratories Inc., Burlingame, California, USA) and HistoMark Orange (Kirkegaard and Perry Laboratories Inc., Gaithersburg, Maryland, USA) were used as substrate and amplifier, respectively. Detection of $\operatorname{cd} 31$ staining was performed using mouse anti-cd31 antibody (Santa Cruz Biotechnology Inc., Santa Cruz, California, USA) and an EnVision staining kit (DAKO Corp., Carpinteria, California, USA). Slides were examined under a bright-field microscope (Nikon Inc., Melville, New York, USA) and images were analyzed using NIH Scion Image software (Scion Corp., Frederick, Maryland, USA).

In the rat hindlimb ischemic model, capillary vascularization was determined by density of vWF- and cd31positive vessels and those filled with latex (averaged from three muscle sections with six fields per section). In murine hindlimb ischemic models, capillary vascularization was measured by density of latex-filled capillaries. In NPY transgenic (NPY-Tg) rats, capillary vascularization of nonischemic muscles was determined by counting the number of capillaries per field in four to six fields per muscle and calculating the number of capillaries per fiber. Collateralization in ischemic hindlimb was measured by counting the number of corkscrew-like, thick-walled vessels per field, averaged from four to six fields per muscle section (averaged from three sections taken at $3 \mathrm{~mm}$ above, $3 \mathrm{~mm}$ below, and at ligation level).

Blood flow measurement. Tissues were weighed and incubated overnight with Digest Reagent (Interactive Medical Technologies Inc.). Ultraspheres were collected according to the manufacturer's directions and counted under the microscope (Nikon Inc.) using improved Neubauer ruling. Tissue blood flow $(\mathrm{ml} / \mathrm{min} / \mathrm{mg})$ was calculated as the number of Ultraspheres collected multiplied by the known flow in the kidney $(\mathrm{ml} / \mathrm{min} / \mathrm{mg})$, divided by the known number of Ultraspheres injected. Aortic ring sprouting assay. Aortic rings were prepared from murine thoracic and abdominal aorta as previously described (29). Briefly, transverse aortic sections (1-2 $\mathrm{mm}$ ) were embedded in Matrigel in eight-well chamber slides and incubated in DMEM containing $10 \% \mathrm{FBS}$ at $37^{\circ} \mathrm{C}$ overnight. Then $1 \%$ endothelial cell growth supplement (Sigma-Aldrich) was added. Once sprouting began (normally in 3-5 days), the medium was changed to medium 199 with or without drugs and incubation continued for another 2-3 days. Then sprouts were fixed, stained, and photographed using the Nikon E600 digital image system and images were analyzed and quantified using NIH Scion Image software (Scion Corp.) and Adobe Photoshop.

RT-PCR of NPY, NPY receptors, and DPPIV mRNA's. Relative, semiquantitative RT-PCR was performed using $18 \mathrm{~S}$ rRNA as an internal control added to each reaction. First-strand cDNA was synthesized from total RNA by MMLV reverse transcriptase (Stratagene, La Jolla, California, USA) using random primers. The cDNA's were amplified by PCR for 30 cycles $\left(94^{\circ} \mathrm{C}\right.$ for
1 minute, $52^{\circ} \mathrm{C}$ for 1 minute 20 seconds, and $72^{\circ} \mathrm{C}$ for 1 minute 15 seconds) using Taq DNA polymerase (Promega Corp., Madison, Wisconsin, USA). The reactions were carried out in the presence of the following pairs of specific primers. NPY: $5^{\prime}$-TACCCCTCCAAGCCGGACAA-3' and $5^{\prime}$-TCTCATTTCCCATCACCACATG-3'; Y1: 5'-CTCTTGCTTATGGRGATGTGA-3' and 5'-CTGGAAGTTTTTGTTCAGGAAYCCA-3'; Y2: 5'-CCTACTGCTCCATCATCTTGC-3' and 5'-GTAGTTGCTGTTCATCCAGCC-3'; Y5: 5'-ATGGAGTTTAAGCTTGAGGAGC-3' and 5'-TGTGTAGGCAGTGGATAAGG G-3'; DPPIV: 5'-GTCCTGGAGGACAATTCTGC-3' and $5^{\prime}$-TGGAGATCTGAGCTGACTGC$3^{\prime}$; and 18S rRNA: 5'-TCAAGAACGAAAGTCGGAGG-3' and $5^{\prime}$-GGACATCTAAGGGCATCACA-3'. PCR products were electrophoresed on a $2 \%$ agarose gel and visualized by ethidium bromide.

Human endothelial cells. ECV cells (a gift of S. Hughes, St. Thomas Hospital, London, United Kingdom) were cultured in DMEM containing 10\% FBS, $2 \mathrm{mmol} / \mathrm{l}$ glutamine, $1,000 \mathrm{U} / \mathrm{dl}$ penicillin, $100 \mu \mathrm{g} / \mathrm{ml}$ streptomycin, and $15 \mathrm{mM}$ HEPES buffer (BioFluids Inc., Rockville, Maryland, USA). Cells were subcultured when $90 \%$ confluent and made quiescent by 48 hours of incubation in $0.5 \%$ FBS-containing medium. Then the indicated drugs were added to the same medium for an additional 24 hours.

Materials. NPY was purchased from Peninsula Laboratory Inc. (Belmont, California, USA), $\mathrm{VEGF}_{121}$, anti-fetal liver kinase 1 antibody (anti-flk1) antibody and bFGF from R\&D Systems Inc. (Minneapolis, Minnesota, USA), Matrigel from Becton, Dickinson and Co. (Bedford, Massachusetts, USA), and sodium nitroprusside and L-NAME were from Alexis Co. (San Diego, California, USA). Y1 antagonist $\mathrm{H} 409 / 22$ and its inactive enantiomer, H510/445, were gifts from AstraZeneca.

Statistical analysis. The SigmaStat (SPSS Science, Chicago, Illinois, USA) was used to perform one-way repeated measures ANOVA followed by the post-hoc Dunnett $t$ test were used for all statistical analysis $(P<0.05$ was considered significant). Data are presented as mean \pm SEM and were graphed using SigmaStat, Microsoft Excel, and Origin 5.0 software (OriginLab, Northampton, Massachusetts, USA).

\section{Results}

Effects of ischemia on plasma NPY levels and expression of NPY receptors and DPPIV in rat hindlimb. Fourteen days after femoral artery occlusion in rats, femoral venous plasma NPY immunoreactivity levels in the occluded limb were 4.5 -fold higher than in the nonoccluded limb (Figure 1a) or in the abdominal aorta $(0.746 \pm 0.200 \mathrm{pmol} / \mathrm{ml}, P<0.01)$ (not shown). This response was decreased $35 \%$ by ipsilateral lumbar sympathectomy (Figure 1a). Administration of the NPY pellet doubled the ischemia-induced plasma peptide levels (Figure 1a). Femoral artery occlusion also decreased (by 60\%) NPY immunoreactivity levels in the artery below the occlusion compared with the contralateral nonoccluded artery (Figure 1b). The 
NPY immunoreactivity level in the femoral artery below the occlusion was further reduced (to $6-10 \%$ of nonischemic levels) by ipsilateral sympathectomy (Figure 1b), indicating efficacy of denervation.

Ischemia also altered NPY receptor expression (Figure 1c). In nonischemic gastrocnemius muscles of control unoperated and nonischemic contralateral muscles of the operated rats, no Y2 or Y5 receptor or DPPIV expression could be detected by RT-PCR; the only detectable mRNA was that of Y1 and NPY (Figure 1c). Conversely, ischemic muscles showed detectable expression of Y2 and DPPIV mRNA's, and NPY administration also induced Y5 mRNA (Figure 1c).

Capillary angiogenesis and collateral formation in the rat ischemic hindlimb after administration of NPY. NPY was administered locally in a slow-release pellet at a dose of $1 \mu \mathrm{g}$ per pellet delivered over 14 days, which was estimated to yield physiological levels. We also used an antagonist selective for the Y1 receptor, NPY's main vasoconstrictive receptor (26), to block NPY-mediated vasoconstriction and to confirm whether $Y 1$ receptors are angiogenic. Thus, NPY was given to three groups of rats: NPY combined with intra-arterial infusion of the $\mathrm{Y} 1$ antagonist $\mathrm{H} 409 / 22$, its inactive enantiomer $\mathrm{H} 510 / 445$, or vehicle alone.

Angiogenic effects of NPY in ischemic tissues were assessed by evaluating vascularization of gastrocnemius muscles and the footpad. Normal, nonischemic gastrocnemius muscles showed rich density of vessels evident by positive staining with two endothelial markers, vWF and cd31 (Figure 2, a and b). In contrast, ischemic muscles and the footpad were pale and had a tendency for reduced density of vessels stained positively for $\mathrm{vWF}$ and $\mathrm{cd} 31$ (Figure 2, a and b). The angiogenic index, indicating density of perfused vessels (latex-filled, vWF-positive) to total microvascular density (all vWF-positive vessels), was reduced in ischemic gastrocnemius muscle and the footpad (Figure $2 \mathrm{a}, P<0.01$ compared with nonischemic values).

NPY application markedly increased vWF- and cd31positive vessels in ischemic tissues to the levels observed in nonischemic tissues (Figure 2, a and b). The angiogenic index of the ischemic tissues was also restored to normal by NPY in the muscle and the footpad (Figure 2a, $P<0.01$, compared with ischemic values). The Y1 receptor antagonist $\mathrm{H} 409 / 22(0.02 \mu \mathrm{mol} / \mathrm{kg} / \mathrm{min}$ for 14 days) had no effect of its own and also did not significantly alter the NPY effect in ischemic gastrocnemius muscles or the footpad compared with tissues treated with NPY and either placebo or Y1-inactive enantiomer H510/445 (Figure 2, a and b). NPY pellets placed in nonischemic tissues did not augment their vascular density significantly but there was a tendency for an increase (Figure 2b). Also, ipsilateral lumbar sympathectomy alone did not significantly change vascular density of ischemic gastrocnemius muscles (data not shown). This, together with incomplete blockade of ischemia-induced increase in plasma NPY suggests that ipsilateral lumbar sympathectomy may have been insufficient for complete limb denervation (due to crossover of fibers from the contralateral side) or that there are other, non-neurogenic sources of NPY (see Discussion).

To test whether NPY stimulates formation of collateral vessels, we analyzed arterial densities in the adductor
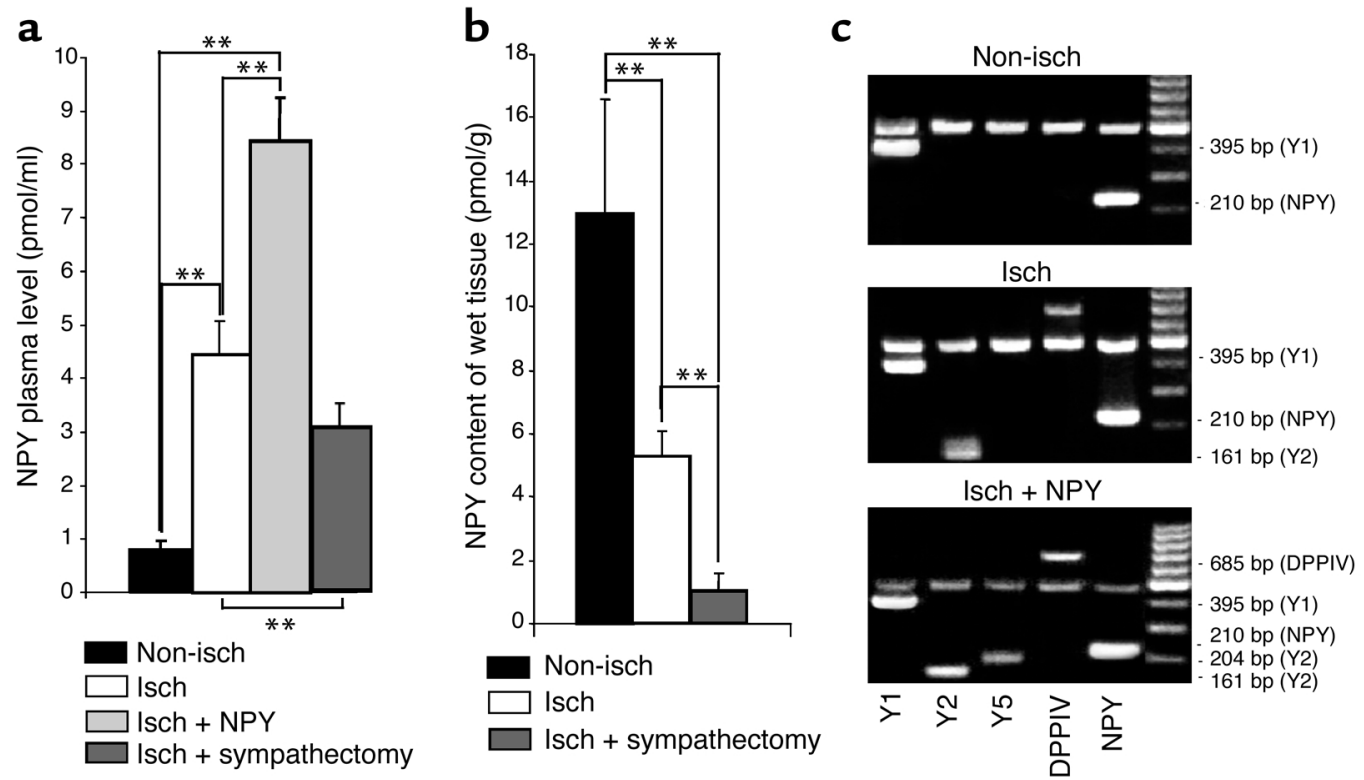

Figure 1

Rat hindlimb ischemia stimulates NPY release and upregulates its receptors and DPPIV. (a) Femoral venous NPY immunoreactivity levels in rat ischemic (isch) and contralateral nonischemic (non-isch) hindlimbs ( $n=10$ each) and in the ischemic limb with ipsilateral lumbar sympathectomy $(n=4)$. (b) Ischemia- and sympathectomy-induced depletion of the NPY immunoreactivity content in the femoral arteries below the occlusion. ${ }^{*} P<0.01$. (c) Ischemia-induced upregulation of expression of Y2 receptor and DPPIV, and NPY-mediated induction of Y5 receptor mRNA in gastrocnemius muscles (semiquantitative RT-PCR). 
a

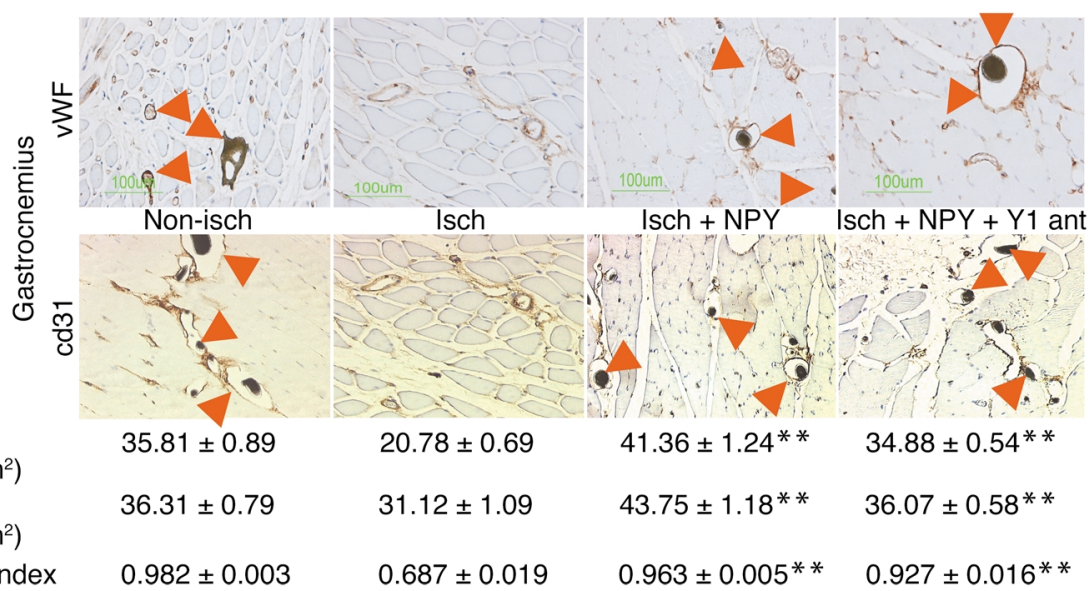

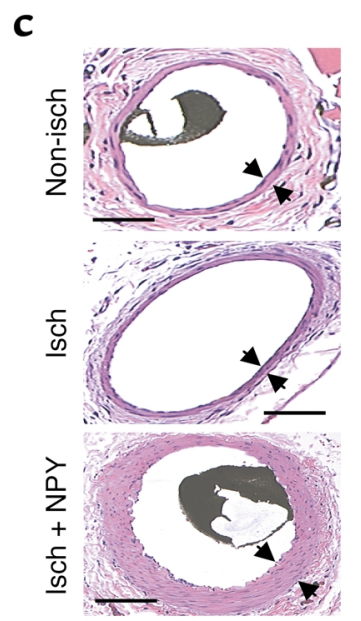

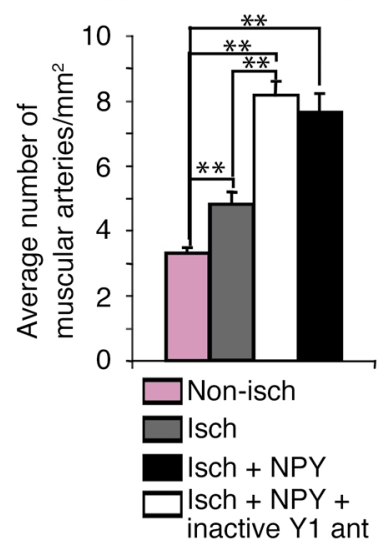

\section{Figure 2}

NPY stimulates capillary angiogenesis (a and $\mathbf{b})$ and collateral formation (c) in rat ischemic hindlimb. (a) Density of vWF- and cd31-positive (a measure of total microvascular density), and latex-filled (a measure of vessels perfused, red arrowheads) vessels in ischemic and nonischemic gastrocnemius muscles $(\times 100)$. Angiogenic index is a ratio of latex-perfused to total microvascular density. (b) Density of vWF-positive vessels in ischemic and nonischemic tissues with and without NPY, Y1 receptor antagonist (active and inactive), and placebo $\left({ }^{*} P<0.01\right.$ compared with ischemic and placebo group [white bars, $n=10$ ]. (c) Representative vessels in the area around the femoral artery occlusion in the adductor muscle (bars $=20 \mu \mathrm{m}$ ) following perfusion with latex (H\&E staining) and graph showing number of these arteries $/ \mathrm{mm}^{2}(* * P<0.01, n=10)$. Arrows indicate media thickness. Ant, antagonist.

muscle around the site of femoral artery occlusion. Since arteriogenesis requires proliferation of VSMCs and leads to formation of corkscrew-like, thick-walled vessels (30), we used the latter feature as a marker for collateral formation. Femoral artery occlusion alone (in placebotreated limbs) increased density of thick-walled muscular arteries in the adductor muscle compared with the contralateral side. NPY stimulated this process by an additional $42 \%$ (Figure 2c).

Effects of NPY on blood flow and contractile function of ischemic muscles. In gastrocnemius muscle, femoral artery occlusion alone induced a $78 \%$ reduction in blood flow compared with contralateral nonischemic muscles (Figure 3a). In the more distal tissues of the footpad, blood flow was even more reduced, reaching $9.6 \%$ of that in contralateral nonischemic tissues (Figure $3 b)$. NPY (1 $\mu$ g per pellet delivered over 14 days) completely restored blood flow in the ischemic gastrocnemius muscle (Figure 3a) and increased footpad flow up to $50 \%$ (Figure $3 \mathrm{~b}$ ). The NPY responses were resistant to $\mathrm{Y} 1$ receptor antagonist $(\mathrm{H} 409 / 22,0.02$ $\mu \mathrm{mol} / \mathrm{kg} / \mathrm{min}$ for 14 days). The Y1 receptor antagonist also had no effect of its own on blood flow in ischemic tissues (data not shown).

To determine whether NPY improves not only the structure but also ischemic tissue function, we studied the contractile performance and fatigability of the rat ischemic gastrocnemius muscle in response to repeated stimulations of the gastrocnemius nerves with six series of an increasing number of trains (total of 112 stimulations) under isometric conditions. The maximal peak tetanic force of gastrocnemius muscle was significantly reduced in ischemic $(3.6 \pm 0.4 \mathrm{~N})$ compared with nonischemic conditions $(4.8 \pm 0.3 \mathrm{~N}, P<0.05)$ (Figure 3 ), but its value increased toward nonischemic levels with administration of the NPY pellet ( $4.4 \pm 0.4 \mathrm{~N}$, Figure $3 \mathrm{e})$. Ischemia also slowed the rate of development (isometric speed) of the first tetanic contraction (Figure 3f). Over the course of stimulations, there was a progressive decrease in the peak value of contractions (the lowest being the last, 112th contraction) in all groups (Figure $3, \mathrm{c}-\mathrm{e})$, indicating the development of fatigue. The 


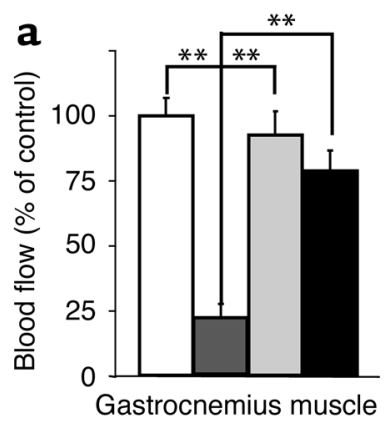

C Tetanic contractions

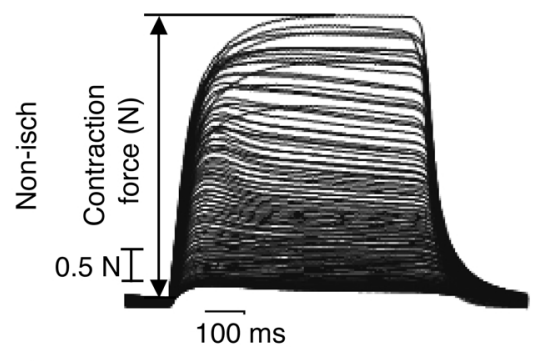

b

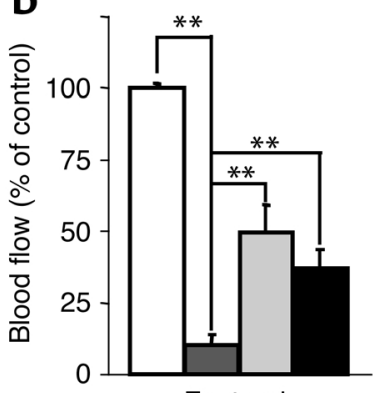

Footpad

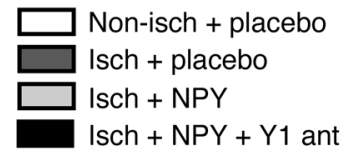

d
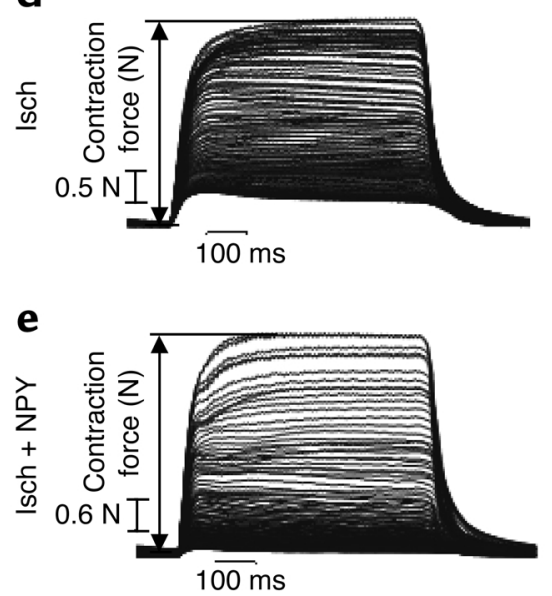

f

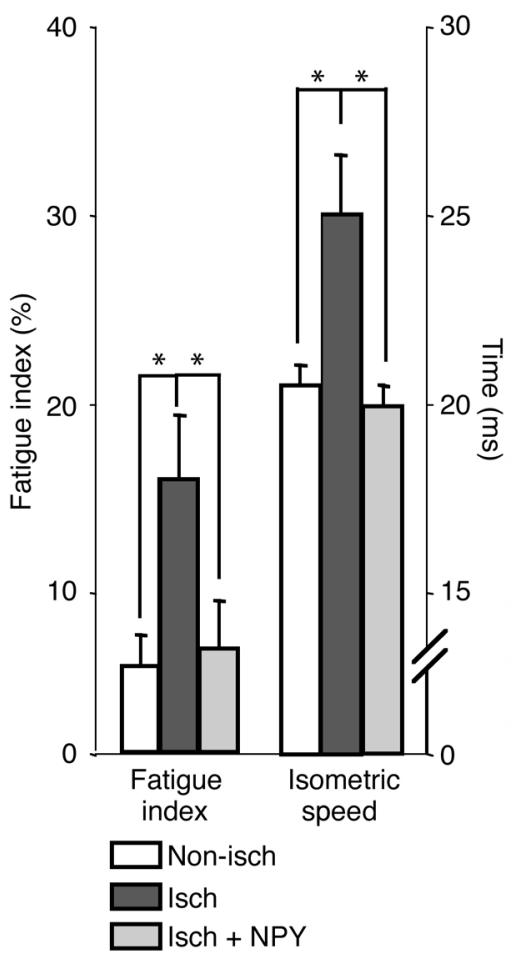

Figure 3

NPY normalizes blood flow of ischemic gastrocnemius muscle (a) and improves it in the footpad (b), and restores function of ischemic gastrocnemius muscle. (c, d, and e) Individual recordings of 112 tetanic contractions, superimposed on each other, in nonischemic, ischemic, and NPYtreated ischemic muscles. Note the peak force of the first (uppermost) and last (lowest curve) contractions, indicating declining force due to prominent fatigue. (f) Isometric speed (time to attain the peak slope of the first tetanic contraction) and the fatigue index (the percent ratio of the peak force of the first to the 52 nd contraction) in nonischemic, ischemic, and NPY-treated ischemic muscles $\left({ }^{*} P<0.001,{ }^{* *} P<0.01, n=6-8\right)$.

fatigue index (determined as the percent ratio of the peak force of the $52 \mathrm{nd}$ contraction to the first tetanic contraction) was increased in ischemic compared with nonischemic muscle (Figure 3f), indicating decreased fatigability of ischemic muscle. Both parameters - isometric speed and fatigue index - were fully normalized by NPY (Figure 3f).

Ischemic and nonischemic angiogenesis in NPY-Tg rats and $N P Y^{-/-}$and $\mathrm{Y}^{-/-}$mice. To test the angiogenic effects of long-term elevation of endogenous levels of NPY, rats overexpressing the NPY gene (NPY-Tg mice) under the control of the natural promoter (31) were examined. NPY-Tg rats had markedly increased vascularization of gastrocnemius muscles (Figure 4, a and b). At 22 months of age, skeletal muscles of the NPY-Tg rats possessed a threefold greater number of capillaries per muscle fiber (Figure $4 \mathrm{e}, P<0.01$ ) and $30 \%$ greater muscle fiber area (data not shown) than muscles from WT rats.

In contrast with NPY-Tg rats, $Y 2^{-/-}$mice had a $30 \%$ lower vascular density in nonischemic gastrocnemius muscles than WT $\left(Y^{+/+}\right)$mice had (Figure $4 \mathrm{e}, P<0.01$ ). Like in WT rats, in $\mathrm{Y2}^{+/+}$mice, limb ischemia markedly (73\%) decreased vascular density of the latex-filled vessels in gastrocnemius muscles (Figure 4, c-e, $P<0.01$ ).
In $\mathrm{Y}^{-/-}$mice, this decrease became even more pronounced, and vascular density of ischemic muscle in these mice remained $58 \%$ lower than that of $Y 2^{+/+}$mice (Figure $4 \mathrm{e}, P<0.01$ ).

The $Y 2^{-/-}$mice also showed markedly diminished (by $76 \%$ ) NPY-induced aortic sprouting ex vivo compared with that of $Y^{+/+}$aortas (Figure 4f, $P<0.01$ ). Aortic sprouting was even more impaired in $\mathrm{NPY}^{-/-}$mice compared with WT 129SV mice (Figure 4f). While WT murine aortas began to sprout within 3-5 days, those from $N P Y^{-/-}$mice took 7-10 days to sprout and their spontaneous sprouting was markedly diminished compared with WT mice (Figure 4f, $P<0.01$ ). NPY administration restored aortic sprouting of $\mathrm{NPY}^{-/-}$aortic rings to the same level as that of the WT mice (Figure 4f).

$V E G F$ and NO in NPY-mediated angiogenesis. To test whether NPY's angiogenic activity is mediated by release of VEGF and NO, and if so, whether these factors alter NPY receptor expression, we first used NO donors or inhibitors of NOS in a human endothelial cell line, ECV, which has undetectable NPY receptor expression when quiescent (Figure 5a, control). In ECV cells, NPY induced/upregulated expression of both Y1 and Y2 receptor mRNA as shown by RT-PCR. These effects were mimicked by sodium nitroprusside, an NO 
donor, and inhibited by $1 \mu \mathrm{M}$ L-NAME (Figure 5a). Moreover, NPY-induced aortic sprouting was completely eliminated in $e \mathrm{NOS}^{-/-}$mice (Figure 5, b-d), similar to that of VEGF-treated mice (Figure $5 \mathrm{~d}$ ). This dramatic effect was not assay-specific since deficiency in eNOS also blocked in vivo NPY-mediated capillary tube formation on Matrigel (data not shown).

NPY-induced aortic sprouting in WT mice was also markedly inhibited by a neutralizing anti-flk 1 antibody $(20 \mathrm{ng} / \mathrm{ml})(32)$ (Figure $5, \mathrm{e}-\mathrm{j}, P<0.01)$. Interestingly, anti-flk1 antibody did not completely block, but reduced by $20 \%$, NPY-induced increase in cellular density in the area of the aortic rings, indicating that cell proliferation and migration was affected much less (Figure 5j).

\section{Discussion}

The hypothesis that NPY is a physiological mediator of angiogenesis in vivo was tested in pathophysiological conditions where both neovascularization and sympathetic nerve activity are stimulated, i.e., in ischemia. Both angiogenesis and NPY levels are known to be the highest early in development (33), coinciding with organogenesis and growth, and they decline with age (34). Also, both become activated during myocardial ischemia (35), exercise, and hypoxia (36). Here we demonstrate for the first time to our knowledge, using a variety of models, that NPY is an endogenously active neurogenic factor stimulating ischemic angiogenesis via Y $2 / Y 5$ receptor- and VEGF/NO-dependent pathways.
Ischemia stimulates NPY release and upregulates NPY receptor expression. In rats, femoral artery occlusioninduced limb ischemia increases local venous outflow and lowers femoral arterial content of NPY, indicating its release from vascular stores. This release is at least in part neurogenic since it is decreased by ipsilateral lumbar sympathectomy. The remaining sympathectomy-resistant NPY release may be due to the incompleteness of one-sided denervation, as well as NPY release from the endothelium, which was previously shown to synthesize its own NPY and to internalize sympathetically derived NPY (21).

A major shift occurs in the Y2/Y5 receptor system during ischemia. While nonischemic muscles express only Y1 receptor (and NPY) mRNA's, ischemic muscles show an induction/upregulation of $\mathrm{Y} 2$ receptors and DPPIV, an enzyme forming Y2/Y5-selective agonist (24). These effects are further augmented by the NPY pellet, which also induces Y5 mRNA. Similar NPY-dependent positive regulation of its own receptors was previously found in VSMCs in vitro $(20,21)$, and in the present study is seen in endothelial cells. Although the exact mechanisms of NPY's upregulation of its own receptors remain unclear, $\mathrm{NO}$ appears to play a role. We now show that an NO donor increases and an NOS inhibitor reduces both NPYmediated upregulation of receptors and endothelial cell proliferation (E.W. Lee et al., unpublished data). Moreover, in $\mathrm{eNOS}^{-/-}$mice, NPY-induced aortic sprouting is completely abolished, as is that of VEGF (37).
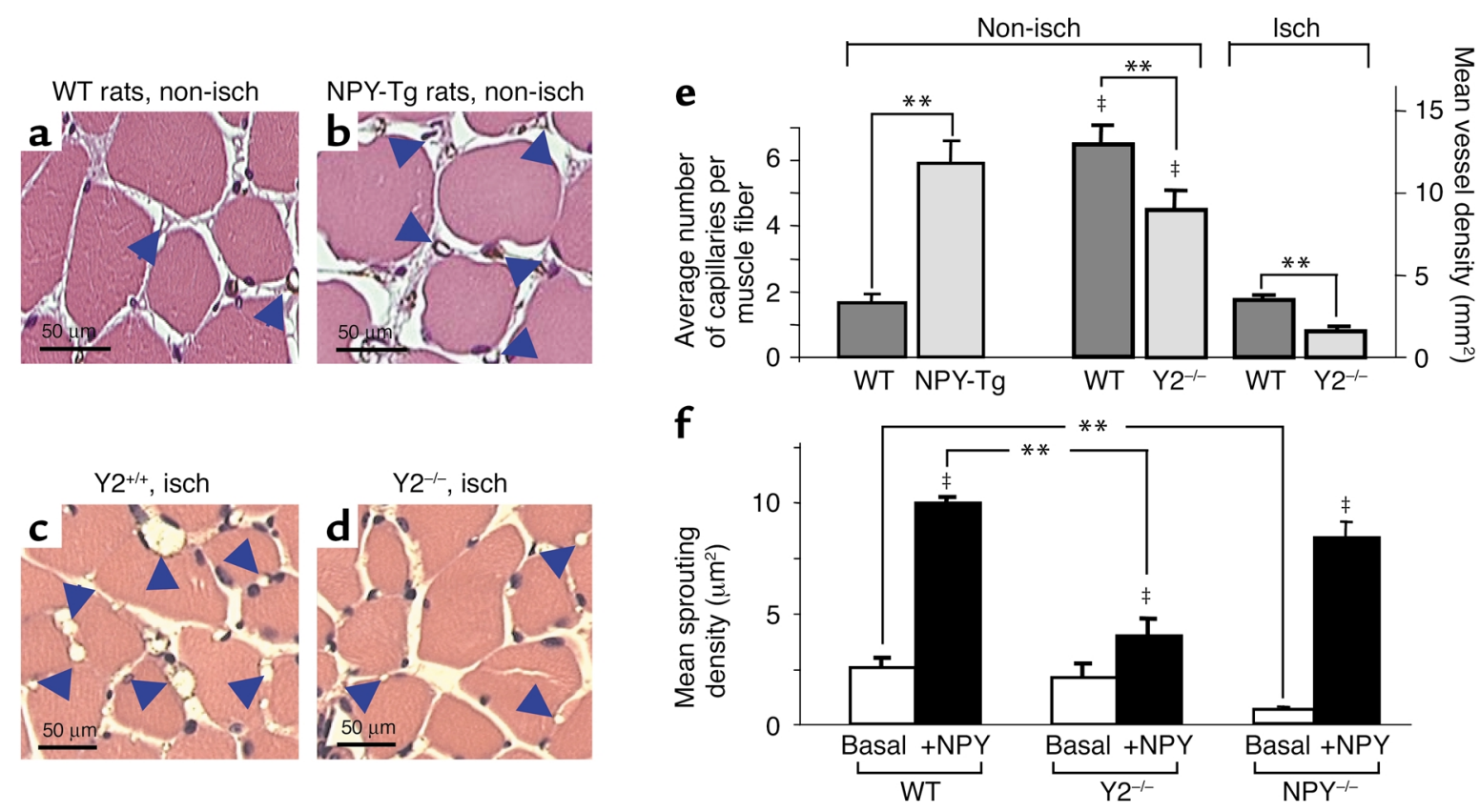

\section{Figure 4}

Vascularization is increased in nonischemic muscles of NPY-Tg rats ( $\mathbf{a}, \mathbf{b}$, and $\mathbf{e})$ and reduced in ischemic and nonischemic muscles of $Y 2^{-/-}$ mice (c-e). (a, b, and e) Vascularization of nonischemic gastrocnemius muscles in 22-month-old NPY-overexpressing rats (NPY-Tg, ${ }^{*} P<0.01$, $n=6-8)$. (c-e) Reduced vascularization (measured by latex-filled capillaries, blue arrowheads) of nonischemic and ischemic gastrocnemius muscles of $Y 2^{+/+}$and $Y 2^{-/-}$mice $\left({ }^{*} P<0.01\right.$ and $\neq P<0.01$ compared with corresponding WT and $Y 2^{-/-}$mice in ischemic group, $\left.n=6\right)$. (f) Inhibition of spontaneous and/or NPY-induced aortic sprouting in $Y 2^{-/-}$and $N P Y^{-/-}$mice compared with WT mice $\left({ }^{* *} P<0.01\right.$ and $\ddagger P<0.01$ compared with corresponding basal group, $n=4-8)$. 
a
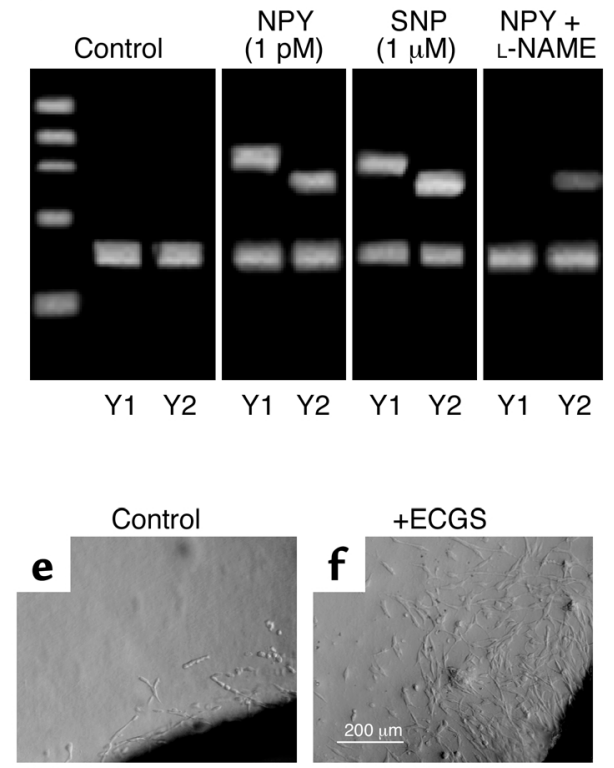

NPY (10 ng/ml)

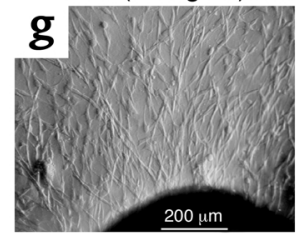

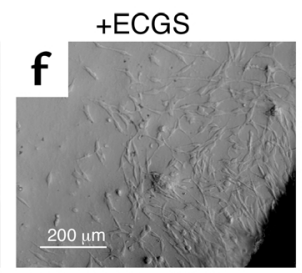

NPY + anti-flk1

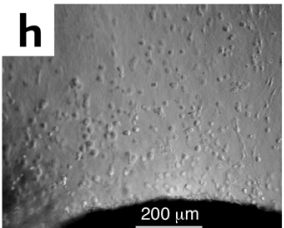

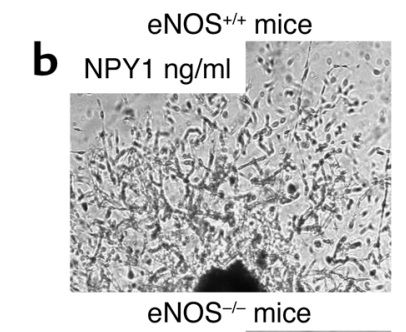

C $\mathrm{NPY} 1 \mathrm{ng} / \mathrm{ml}$

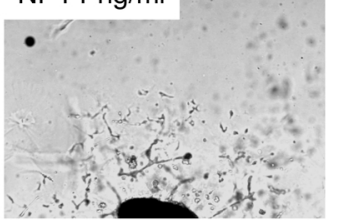

i

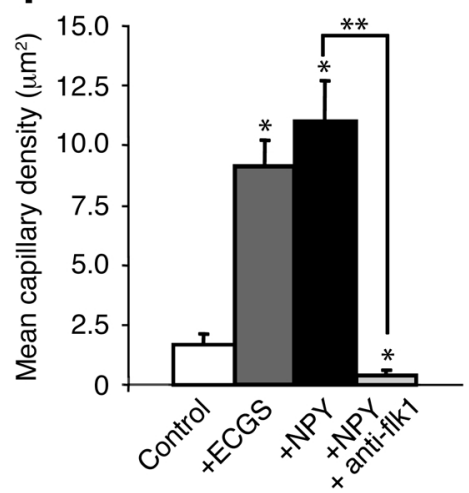

d

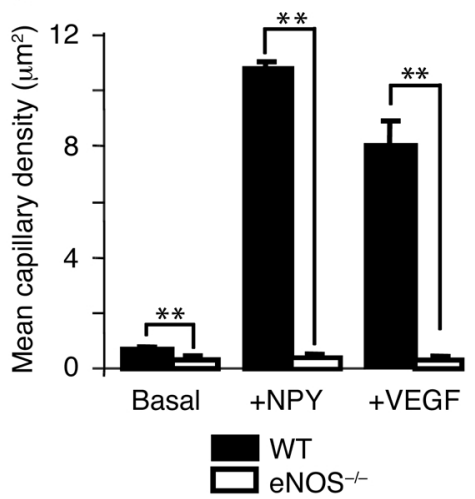

j

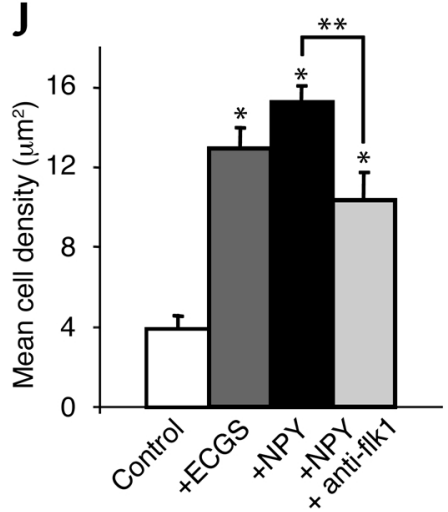

Figure 5

NPY-induced angiogenesis is mediated by eNOS and VEGF. (a) Semiquantitative RT-PCR showing NPY-and sodium nitroprusside-induced upregulation of NPY receptors and inhibition of receptors by L-NAME in human endothelial cell line ECV. (b-d) Inhibition of basal-, NPY-, and VEGF-induced sprouting in $e N^{-1-}$ aortas $\left({ }^{*} P<0.001, n=6-8\right)$. (e-j) Inhibition of NPY-induced aortic sprout formation but not cell migration/proliferation (mean cell density) by anti-flk1 antibody $\left({ }^{*} P<0.001\right.$ and ${ }^{*} P<0.01$ compared with control [white bars] $\left.n=8\right)$. SNP, sodium nitroprusside. ECGS, endothelial cell growth supplement (Sigma-Aldrich).

Thus, endothelial NO appears to be essential for NPY's angiogenic signaling, in part due to its upregulation of NPY receptor expression.

Administration of NPY revascularizes and restores blood flow to ischemic tissues. When administered locally to the rat ischemic hindlimb, NPY stimulates angiogenesis and restores vascularization of ischemic tissues to nonischemic levels. This occurs at physiological plasma NPY concentrations $(<10 \mathrm{nM})$, which may be vasoconstrictive, similar to those induced by stress or exercise in rats $(19,26)$. There is no indication, however, that NPYmediated vasoconstriction occurs in these conditions. First, NPY-induced angiogenesis is accompanied by the restoration of blood flow to the ischemic gastrocnemius muscles and a 50\% improvement of flow to the footpad. Second, both structural and functional NPY-mediated improvements are resistant to the $\mathrm{Y} 1$-selective receptor antagonist (H402/22), indicating that Y1-mediated vasoconstriction does not play a role and that $\mathrm{Y} 1$ receptors are not angiogenic in the ischemic hindlimb.

The possibility, however, that NPY may exert an angiogenic effect in nonischemic tissues by activating Y1 receptors is suggested by the tendency for NPY to increase vascularization of the nonischemic muscle, which expresses only this receptor type (as shown by RT-PCR). Also our previous findings of the ability of Y1 receptor antagonist to reduce already markedly impaired aortic sprouting in $Y 2^{-/-}$mice (38) suggest that $\mathrm{Y} 1$ receptors play a compensatory role in the absence of the major angiogenic $\mathrm{Y} 2$ receptor.

NPY also stimulates formation of collateral vessels in the adductor muscle at the level of the occluded femoral artery. This process, called arteriogenesis, differs from capillary angiogenesis by being hypoxia-independent but shear stress-dependent (30), and also requires proliferation of VSMCs (39). The newly formed collateral arteries often assume corkscrew shapes $(39,40)$, possibly due to the nonuniformity of their VSMC proliferation and formation of hypertrophied media. NPY, due to its potent growth-promoting activities for VSMCs (20) and endothelial cells (21), stimulates both collateralization and capillary angiogenesis, leading to the full revascularization of ischemic tissues. Although stimulation of VSMC proliferation is necessary for collateral formation and salvaging of ischemic tissue, it may also carry the risk of 
promoting neointima formation and atherosclerosis. Such concerns have been raised for several angiogenic factors, such as VEGF and bFGF (41), and may also apply to NPY. However, although NPY promotes angioplasty-induced neointima formation in rat carotid artery, its effects appear to be mediated by Y1, and not Y2, receptors (L. Li et al., unpublished data).

The loss of the angiogenic influence of NPY may explain the long-term ineffectiveness of regional sympathectomy in patients with severe peripheral vascular insufficiency (42) when it is used to alleviate ischemic pain. Although in our studies sympathectomy did not significantly reduce ischemic revascularization, this could be due to the short observation time ( 2 weeks) and incomplete removal of NPY by unilateral sympathectomy (endothelial NPY and/or crossover of contralateral fibers).

Administration of NPY restores ischemic muscle contractile function. Along with blood flow, NPY restores the contractile performance of the ischemic gastrocnemius muscle. Ischemia led to several impairments of muscle function, such as a decrease in maximal peak force of tetanic contraction and slower development of peak contraction (isometric speed). In contrast, the fatigability of the ischemic muscles in response to long series of tetanic stimulations decreased compared with nonischemic muscles. Such changes have been reported previously (43) and are believed to be due to the muscle's selective loss of ischemia-sensitive fast glycolytic fibers and its switch to fast-oxidative ones, which are less fatigued (44). NPY increased the maximal peak force of tetanic contraction of the ischemic gastrocnemius muscle toward normal and fully restored its isometric speed of contraction and fatigability. These data demonstrate the peptide's ability to restore not only ischemic tissue vascularization and blood flow but also its contractile function.

Endogenous NPY and Y2 receptors are angiogenic. To test whether the angiogenic activity of NPY plays a longterm role during tissue growth and development, we used rats overexpressing the NPY gene under the control of the natural promoter $(45,46)$. These rats, which do not have elevated plasma NPY at rest but have slightly elevated tissue NPY levels (31), have a remarkable hypervascularity of gastrocnemius muscles at 5, 12 (data not shown), and 22 months of age, suggesting enhanced angiogenesis during their lifelong skeletal muscle growth. This hypervascularization appears to be functional as it is associated with skeletal muscle hypertrophy (data not shown), lower blood pressure, and increased longevity of these rats (45). Chronically elevated NPY levels may also protect against the age-dependent decline in angiogenesis. Previously we noted that aging impairs NPY angiogenic activity in vitro and also inhibits expression of Y2 receptors and DPPIV (47). In NPY-Tg rats, NPYinduced protection against declining angiogenesis could be due to the peptide's ability to upregulate its own angiogenic receptors (20).
The primary receptor involved in NPY's angiogenic actions in ischemia and during developmental growth appears to be Y2. Removal of Y2 receptors by gene knockout reduces vascularization by $30-60 \%$ in both nonischemic and ischemic gastrocnemius muscles compared with corresponding muscles of $\mathrm{Y2}^{+/+}$ mice. Similarly, Y2 receptor knockout and the Y2antagonist treatment of $\mathrm{Y2}^{+/+}$aortas (38) markedly inhibit NPY-mediated aortic sprouting ex vivo. The role of Y5 receptor cannot be similarly assessed due to the lack of data on $\mathrm{Y5}^{-/-}$mice (investigation in process). In the in vitro aortic sprouting assay, however, NPY-mediated angiogenic effect in the WT aortas is blocked by $\mathrm{Y} 5$ receptors, while in the $Y 2^{-/-}$mice, Y1 receptors may also play a compensatory angiogenic role (38). The latter notion is also supported by near-normal spontaneous aortic sprouting in $\mathrm{Y}^{-/-}$ mice. However, knockout of the NPY gene itself severely impairs spontaneous sprouting of murine aortas, which administration of NPY fully restores. These data indicate that endogenous NPY is a required growth factor for stimulation of angiogenesis, at least in the model of murine aorta.

NPY-mediated angiogenesis is VEGF- and NO-dependent. Having determined potent mitogenic activities of NPY on VSMCs and endothelial cells in vitro and its angiogenic activities in vivo, we also sought to establish mechanisms of NPY's angiogenesis. There are many mediators and signaling pathways that lead to angiogenesis (4). Some growth factors such as VEGF stimulate neovascularization partly by releasing $\mathrm{NO}$, which by itself may be angiogenic (37). NO also appears to be involved in NPY's actions: in cerebral vessels, it mediates NPY-induced vasodilation, which is Y2 receptor-dependent (48). In support of the role of VEGF, NPY-mediated aortic sprouting was blocked by an antibody neutralizing a VEGF receptor, flk1 (32). However, in spite of this dramatic effect, antiflk1 antibody only slightly reduced the density of endothelial cells migrating out of the rings. This indicates that VEGF is not involved in NPY's effect on cell migration and/or proliferation but mediates the later stage of angiogenesis, endothelial cell differentiation into capillaries. In contrast, deficiency of eNOS completely prevented all components of NPY-induced angiogenic response in aortic sprouting assays, implying that NO is required for full elaboration of NPYmediated angiogenesis.

In conclusion, NPY is an endogenously active neurogenic (and possibly endothelial) ischemia-induced angiogenic factor that stimulates ischemic and growth-dependent angiogenesis by activating Y2/Y5 receptors and NO-dependent pathways, in part mediated by release of VEGF. Exogenous NPY, at physiological concentrations, restores not only vascularization but also the function of the ischemic tissues. Thus, NPY-derived drugs (Y2/Y5 agonists) might be therapeutic for revascularization of ischemic tissues in peripheral vascular and heart diseases. 


\section{Acknowledgments}

This study was supported in part by NIH grants HL-67357 (to Z. Zukowska) and HL-57921 (to M. Michalkiewicz).

1. Risau, W. 1997. Mechanisms of angiogenesis. Nature. 386:671-674.

2. Baffour, R., et al. 1992. Enhanced angiogenesis and growth of collaterals by in vivo administration of recombinant basic fibroblast growth factor in a rabbit model of acute lower limb ischemia: dose-response effect of basic fibroblast growth factor. J. Vasc. Surg. 16:181-191.

3. Shweiki, D., Itin, A., Soffer, D., and Keshet, E. 1992. Vascular endothelial growth factor induced by hypoxia may mediate hypoxia-initiated angiogenesis. Nature. 359:843-845.

4. Carmeliet, P. 2000. Mechanisms of angiogenesis and arteriogenesis. Nat. Med. 6:389-395.

5. Gargett, C.E., and Rogers, P.A. 2001. Human endometrial angiogenesis. Reproduction. 121:181-186.

6. Egginton, S., et al. 1998. Capillary growth in relation to blood flow and performance in overloaded rat skeletal muscle. J. Appl. Physiol. 85:2025-2032.

7. Kerbel, R.S. 2000. Tumor angiogenesis: past, present and the near future. Carcinogenesis. 21:505-515.

8. Breier, G., Damert, A., Plate, K.H., and Risau, W. 1997. Angiogenesis in embryos and ischemic diseases. Thromb. Haemost. 78:678-683.

9. Folkman, J. 1995. Angiogenesis in cancer, vascular, rheumatoid and other disease. Nat. Med. 1:27-31.

10. Ware, J.A., and Simons, M. 1997. Angiogenesis in ischemic heart disease. Nat. Med. 3:158-164.

11. Isner, J.M. 1999. Manipulating angiogenesis against vascular disease. Hosp. Pract. (Off. Ed.) 34:69-74, 76, 79-80 passim.

12. Rivard, A., and Isner, J.M. 1998. Angiogenesis and vasculogenesis in treatment of cardiovascular disease. Mol. Med. 4:429-440.

13. Folkman, J. 1998. Therapeutic angiogenesis in ischemic limbs. Circulation. 97:1108-1110

14. Ikeda, E., Achen, M.G., Breier, G., and Risau, W. 1995. Hypoxiainduced transcriptional activation and increased mRNA stability of vascular endothelial growth factor in C6 glioma cells. J. Biol. Chem. 270:19761-19766.

15. Isner, J.M., and Asahara, T. 1999. Angiogenesis and vasculogenesis as therapeutic strategies for postnatal neovascularization. J. Clin. Invest. 103:1231-1236.

16. Mack, C.A., et al. 1998. Biologic bypass with the use of adenovirus-mediated gene transfer of the complementary deoxyribonucleic acid for vascular endothelial growth factor 121 improves myocardial perfusion and function in the ischemic porcine heart. J. Thorac. Cardiovasc. Surg. 115:168-176; discussion 176-177.

17. Sellke, F.W., Laham, R.J., Edelman, E.R., Pearlman, J.D., and Simons, M. 1998. Therapeutic angiogenesis with basic fibroblast growth factor: technique and early results. Ann. Thorac. Surg. 65:1540-1544.

18. Bevan, R.D. 1984. Trophic effects of peripheral adrenergic nerves on vascular structure. Hypertension. 6:III19-III26.

19. Zukowska-Grojec, Z., and Wahlestedt, C. 1993. Origin and actions of neuropeptide $Y$ in the cardiovascular system. In The biology of neuropeptide $Y$ and related peptides. W.F. Colmers and C. Wahlestedt, editors. Humana Press. Totowa, New Jersey, USA. 315-388.

20. Zukowska-Grojec, Z., Karwatowska-Prokopczuk, E., Fisher, T.A., and Ji, H. 1998. Mechanisms of vascular growth-promoting effects of neuropeptide Y: role of its inducible receptors. Regul. Pept. 75-76:231-238.

21. Zukowska-Grojec, Z., et al. 1998. Neuropeptide Y: a novel angiogenic factor from the sympathetic nerves and endothelium. Circ. Res. 83:187-195.

22. Ingenhoven, N., and Beck-Sickinger, A.G. 1999. Molecular characterization of the ligand-receptor interaction of neuropeptide Y. Curr. Med. Chem. 6:1055-1066.

23. Ghersi, G., Chen, W., Lee, E.W., and Zukowska, Z. 2001. Critical role of dipeptidyl peptidase IV in neuropeptide Y-mediated endothelial cell migration in response to wounding. Peptides. 22:453-458.

24. Mentlein, R., Dahms, P., Grandt, D., and Kruger, R. 1993. Proteolytic processing of neuropeptide $Y$ and peptide YY by dipeptidyl peptidase IV. Regul. Pept. 49:133-144.

25. Doods, H.N., et al. 1995. Pharmacological characterization of the selective nonpeptide neuropeptide Y Y1 receptor antagonist BIBP 3226 J. Pharmacol. Exp. Ther. 275:136-142.

26. Zukowska-Grojec, Z., Dayao, E.K., Karwatowska-Prokopczuk, E., Hauser G.J., and Doods, H.N. 1996. Stress-induced mesenteric vasoconstriction in rats is mediated by neuropeptide Y Y1 receptors. Am. J. Physiol. 270:H796-H800

27. van Dielen, F.M., et al. 1998. Effects of surgical sympathectomy on skin blood flow in a rat model of chronic limb ischemia. World J. Surg. 22:807-811.

28. Pettorossi, V.E., Della Torre, G., Bortolami, R., and Brunetti, O. 1999. The role of capsaicin-sensitive muscle afferents in fatigue-induced modulation of the monosynaptic reflex in the rat. J. Physiol. 515:599-607.

29. Nicosia, R.F., and Ottinetti, A. 1990. Growth of microvessels in serumfree matrix culture of rat aorta. A quantitative assay of angiogenesis in vitro. Lab. Invest. 63:115-122.

30. Hershey, J.C., et al. 2001. Revascularization in the rabbit hindlimb: dissociation between capillary sprouting and arteriogenesis. Cardiovasc. Res. 49:618-625.

31. Michalkiewicz, M., and Michalkiewicz, T. 2000. Developing transgenic neuropeptide Y rats. In Neuropeptide Y protocols. A. Balasubramaniam, editor. Humana Press. Totowa, New Jersey, USA. 73-89.

32. Conn, G., et al. 1990. Amino acid and cDNA sequences of a vascular endothelial cell mitogen that is homologous to platelet-derived growth factor. Proc. Natl. Acad. Sci. U. S. A. 87:2628-2632.

33. Gordon, L., et al. 1993. Development of the peptidergic innervation of human heart. J. Anat. 183:131-140.

34. Swift, M.E., Kleinman, H.K., and DiPietro, L.A. 1999. Impaired wound repair and delayed angiogenesis in aged mice. Lab. Invest. 79:1479-1487.

35. Morris, M.J., et al. 1986. Plasma neuropeptide Y levels rise in patients undergoing exercise tests for the investigation of chest pain. Clin. Exp. Pharmacol. Physiol. 13:437-440.

36. Kaijser, L., Pernow, J., Berglund, B., Grubbstrom, J., and Lundberg, J.M. 1994. Neuropeptide $Y$ release from human heart is enhanced during prolonged exercise in hypoxia. J. Appl. Physiol. 76:1346-1349.

37. Ziche, M., and Morbidelli, L. 2000. Nitric oxide and angiogenesis. J. Neurooncol. 50:139-148.

38. Lee, E.W., Grant, D.S., Movafagh, S., and Zukowska, Z. 2003. Impaired angiogenesis in neuropeptide $\mathrm{Y}(\mathrm{NPY})$-Y2 receptor knockout mice. Peptides. 24:99-106.

39. Heeschen, C., et al. 2001. Nicotine stimulates angiogenesis and promotes tumor growth and atherosclerosis. Nat. Med. 7:833-839.

40. Deindl, E., et al. 2001. Role of ischemia and of hypoxia-inducible genes in arteriogenesis after femoral artery occlusion in the rabbit. Circ. Res. 89:779-786.

41. Carmeliet, P., and Collen, D. 1997. Molecular analysis of blood vessel formation and disease. Am. J. Physiol. 273:H2091-H2104.

42. Alexander, J.P. 1994. Chemical lumbar sympathectomy in patients with severe lower limb ischaemia. Ulster Med. J. 63:137-143.

43. Lee, S.L., Pevec, W.C., and Carlsen, R.C. 2001. Functional outcome of new blood vessel growth into ischemic skeletal muscle. J. Vasc. Surg. 34:1096-1102.

44. Lieber, R.L., Pedowitz, R.A., Friden, J., and Gershuni, D.H. 1992. Decreased muscle speed, strength and fatigability following two hours of tourniquet-induced ischaemia. Scand. J. Plast. Reconstr. Surg. Hand Surg. 26:127-132.

45. Michalkiewicz, M., Knestaut, K.M., Bytchkova, E.Y., and Michalkiewicz, T. 2003. Hypotension and reduced catecholamines in neuropeptide $Y$ transgenic rats. Hypertension. 41:1056-1062.

46. Michalkiewicz, M., Michalkiewicz, T., Kreulen, D.L., and McDougall, S.J. 2001. Increased blood pressure responses in neuropeptide $Y$ transgenic rats. Am. J. Physiol. Regul. Integr. Comp. Physiol. 281:R417-R426.

47. Kitlinska, J., Lee, E.W., Movafagh, S., Pons, J., and Zukowska, Z. 2002. Neuropeptide Y-induced angiogenesis in aging. Peptides. 23:71-77.

48. You, J., Edvinsson, L., and Bryan, R.M., Jr. 2001. Neuropeptide Y-mediated constriction and dilation in rat middle cerebral arteries. J. Cereb. Blood Flow Metab. 21:77-84. 